\title{
Functionalization of single-walled carbon nanotubes and their binding to cancer cells
}

This article was published in the following Dove Press journal:

International Journal of Nanomedicine

27 February 2012

Number of times this article has been viewed

Seyed Yazdan Madani'

Aaron Tan'

Miriam Dwek ${ }^{2}$

Alexander M Seifalian ${ }^{1,3}$

'UCL Centre for Nanotechnology and Regenerative Medicine,

University College London, London,

UK; ${ }^{2}$ Department of Molecular and

Applied Biosciences, School of Life

Sciences, University of Westminster,

London, UK; ${ }^{3}$ Royal Free Hampstead

NHS Trust Hospital, London, UK

Video abstract

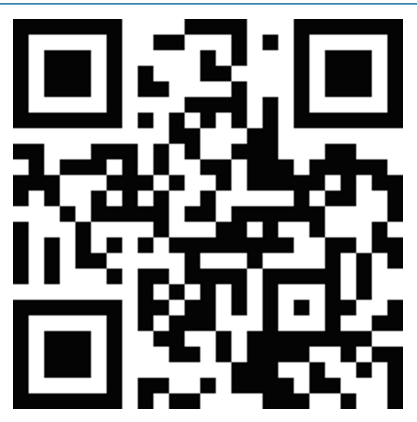

Point your SmartPhone at the code above. If you have a $Q R$ code reader the video abstract will appear. Or use: http://bit.ly/A73evz

Correspondence: Alexander M Seifalian UCL Centre for Nanotechnology and Regenerative Medicine, University College London, London NW3 2QG, UK

Tel +44207830290I

Email a.seifalian@ucl.ac.uk
Background: Single-walled carbon nanotubes (SWCNTs) have novel properties including their nanoscale size and ease of cellular uptake. This makes them useful for drug delivery, and their photo-thermal effects make them potentially useful in a wide range of applications, particularly the treatment of solid tumors. The poor solubility of SWCNTs has, however, been an issue that may potentially limit the utility of SWCNTs for cancer treatment. Functionalization of the surface of the tubes may be an approach to overcome this problem.

Methods: SWCNTs were refluxed in $\mathrm{HNO}_{3} / \mathrm{H}_{2} \mathrm{SO}_{4}(1: 3)$ at $120^{\circ} \mathrm{C}$ for 120 minutes. Transmission electron microscopy (TEM), Fourier transform infrared spectroscopy (FTIR), contact angle measurements, and near infrared (NIR) light exposure were used to assess the functionalization process. The attachment of a carbohydrate-binding protein (lectin) labeled with fluorescein isothiocyanate to the functionalized SWCNTs enabled evaluation of the functionalization step via confocal microscopy. The lectin from Helix pomatia, (Helix pomatia agglutinin [HPA]), can detect changes in protein glycosylation associated with aggressive metastatic cancer. The interaction between the lectin HPA alone and HPA conjugated to the functionalized SWCNTs with human breast cancer cells (MCF-7) was measured using a quartz crystal microbalance biosensor.

Results: Following the functionalization process, TEM images showed a layer had formed on the surface of the SWCNTs. In the FTIR experiment, results illustrated the presence of the $-\mathrm{COOH}$ group on the functionalized SWCNTs. Contact angle measurements showed that upon functionalization the hydrophilicity of the SWCNTs increased. The temperature increase in the liquid (supernatant) surrounding the functionalized SWCNTs following exposure to light in the NIR $(808 \mathrm{~nm})$ was greater than for non-functionalized SWCNTs. The biosensor work showed that HPA binds with high affinity (nanomolar range) to human breast cancer cells; HPA-binding properties to MCF-7 cells were retained following conjugation to the functionalized SWCNTs.

Conclusion: Treating pure SWCNTs with $\mathrm{HNO}_{3} / \mathrm{H}_{2} \mathrm{SO}_{4}(1: 3)$ at $120^{\circ} \mathrm{C}$ for 120 minutes is an effective method for functionalization of SWCNTs. HPA linked to SWCNTs is a suitable candidate for the delivery of the functionalized SWCNTs to cancer cells.

Keywords: SWCNT, HPA lectin, human breast cancer cells

\section{Introduction}

Carbon nanotubes $(\mathrm{CNTs})^{1}$ were discovered more than 50 years ago. Since that time, researchers have unraveled many of the properties of these fascinating materials, and applications now range from the development of nanocomposites, electrochemical devices, electronic devices and sensors, and recently to medicine. ${ }^{2}$ The exceptional tensile strength (in the gigapascal range) and high Young's modulus (in the terapascal range) of CNTs attest to the robust mechanical properties of CNTs. ${ }^{3}$ 
CNTs are mainly cylindrical fullerenes, with dimensions in the nanoscale. ${ }^{4}$ They comprise carbon atoms arranged in a hexagonal lattice. CNTs are found in two forms: as singlewalled (SWCNTs) and as multi-walled carbon nanotubes (MWCNTs). ${ }^{5}$ SWCNTs comprise a single sheet of $\mathrm{sp}^{2}$ hybridized carbon atoms, rolled into a cylindrical format, with a diameter of 0.4-2.0 nm; whilst MWCNTs are made up of concentric layers of SWCNTs, with inner diameters in the range of $1-3 \mathrm{~nm}$ and $2-100 \mathrm{~nm}$ for the outer diameter. ${ }^{6}$

The length of MWCNTs varies greatly depending on their application. The concentric nanotube layers are held together by secondary Van der Waals forces. The walls of each layer of MWCNTs lie parallel to their central axis. ${ }^{7}$ CNTs are available in diverse structural designs and various electron arrangements; due to these structural differences, SWCNT and MWCNT tend to exhibit different physical properties. For example, in terms of their electrical conductivity, SWCNTs may be either semiconductors or conductors, but MWCNTs are only semiconductors. ${ }^{8}$ Furthermore, CNTs have a high optical absorbance in the near infrared (NIR) region ${ }^{9}$ and can readily penetrate cell membranes, ${ }^{10}$ making them ideal as nanoscale "delivery platforms" with unique photothermal properties. Due to these properties, CNTs can be used to both thermally ablate and deliver chemotherapeutic drugs directly into cancer cells. ${ }^{11}$

Following their exposure to NIR light, MWCNTs are less combustible than SWCNTs. ${ }^{12,13}$ The range of properties of CNTs, including their size, high aspect ratio, high surface area, ultra-light weight, and high stability, have meant that these materials have been used in diverse programs of research. ${ }^{4,14}$ CNTs have been exploited in several biomedical applications; for example, for the regeneration of nerve conduits, as scaffolds for the regeneration bone, for the thermal treatment of cancer, and for drug delivery. ${ }^{15}$ It has been shown that by adjusting the size of the CNTs, this will direct them into different cellular pathways, ${ }^{16}$ and also alter their affinity to various ligands, control their uptake by malignant cells, ${ }^{17}$ and potentially minimize adverse effects on normal healthy cells. ${ }^{18}$

Consistent with these observations, renal filtration and nonspecific uptake by the reticuloendothelial system has been shown to be dependent on particle size. Therefore, despite the above advantages of CNTs, their lack of biocompatibility has remained an issue, and this has been recognized as an area where more research is needed. ${ }^{19}$

Pristine CNTs are usually severely contaminated with metal catalysts and amorphous carbons, are generally insoluble, and have adverse effects on the environment of the cell. It has been proposed that the surface functionalization of
CNTs may assist in overcoming these issues. Generally, the functionalization process involves the attachment of materials to the sides or end of the CNTs, hence improving their biocompatibility. ${ }^{20}$ The attachment may be achieved via covalent or noncovalent bonding, ${ }^{21}$ and both systems have their own advantages and disadvantages. For example, covalent bonding of a functional group to the CNTs will produce stable functionalized CNTs, ${ }^{22}$ which would make these materials suitable candidates for different applications such as drug delivery. ${ }^{23}$

However, a problem that may occur with this technique is that the side wall of the CNT may become damaged, resulting in a reduction in some of the other uses of the CNT with respect to Raman scattering and NIR fluorescence signaling. For this reason, it has been suggested that the CNTs functionalized by covalent bonding should not be used in some of the carbon nanotube applications such as imaging. ${ }^{24}$

Noncovalent functionalization has the advantage that it results in the preservation of the electronic structure of the nanotube atomic surface. ${ }^{25-27}$ Attachment of polyethylene glycol (PEG) to the surface of CNTs is a method that improves the solubility ${ }^{28}$ of CNTs. Adding PEG also allows for modification of the CNT with different functional groups such as terminal amine $\left(\mathrm{PEG}-\mathrm{NH}_{2}\right)$ and carboxyl (PEG$\mathrm{COOH})$ groups that offer further new functionalization sites for biomolecules. ${ }^{29}$ PEGylated CNTs demonstrate superior biocompatibility and are currently being assessed for use in medical implants. ${ }^{30}$ Treating CNTs with strong acid is also another common technique used for improving the solubility of these materials. This method leads to the removal of metallic impurities from the CNTs and causes the formation of-COOH groups on their surfaces. ${ }^{30}$ This would be expected to improve the solubility of these materials and to provide a platform for the attachment of other compounds. ${ }^{30}$

The aim of this study was first to investigate the functionalization process of SWCNTs, and secondly, to investigate the attachment of the lectin Helix pomatia agglutinin (HPA) to SWCNTs and to investigate the delivery of SWCNTs to breast cancer cells.

\section{Materials and methods Experimental agents}

SWCNTs were purchased from Nanothinx (Rio-Patras, Greece), HPA lectin-fluorescein isothiocyanate (FITC) $(20 \mu \mathrm{g} / \mathrm{mL})$, 3-dimethylaminopropyl-N-ethylcarbodiimide hydrochloride (EDC) (99\%), $\mathrm{H}_{2} \mathrm{SO}_{4}(95 \%), \mathrm{HNO}_{3}(70 \%), 4 \%$ paraformaldihyde, and N-hydroxysuccinimide (NHS) were purchased from Sigma-Aldrich (Dorset, UK). Dulbecco's modified Eagle's 
medium (DMEM), fetal calf serum (FCS), phosphate buffered saline (PBS), bovine serum albumin (BSA), To-Pro ${ }^{\circledR}-3$, and trypsin $(0.5 \mathrm{~g} / \mathrm{L})$ were purchased from Invitrogen (Paisley, UK). Human breast cancer cell (MCF-7) and human colon adenocarcinoma cell line (SW480) were purchased from ATCC via LGC (Middlesex, UK). Laser diodes of wavelengths $808 \mathrm{~nm}$ were purchased from Thorlabs Ltd (Cambridge, UK) alongside other necessary equipment such as thermoelectric cooler elements and laser diode controller.

\section{Functionalized SWCNTs}

Solid SWCNTs in powder form were purchased from Nanothinx. The purity of SWCNTs used was 97\%. $10 \mathrm{mg}$ of SWCNTs was dispersed in $30 \mathrm{~mL}$ of $1: 3 \mathrm{HNO}_{3}: \mathrm{H}_{2} \mathrm{SO}_{4}$ $\left[\mathrm{HNO}_{3}(70 \%) / \mathrm{H}_{2} \mathrm{SO}_{4}(95 \%-98 \%)\right]$ solution and refluxed for 120 minutes at $120^{\circ} \mathrm{C}$ with stirring at a rate of $500 \mathrm{rpm}$. The acid-treated SWCNTs were centrifuged for 30 minutes at $2400 \times g$ and sonicated for 1 hour. The SWCNTs were washed extensively in deionized water until a constant $\mathrm{pH}$ value in the range of 5-6 was attained.

To investigate the relationship between the exposures to the acid solution of the SWCNTs on the functionalization process, an experiment was set up where the reflux step was repeated for $0.5,1,2,3,4$, and 5 hours. Each sample was subsequently conjugated with HPA-FITC (lectin-FITC). The confocal microscope was utilized to compare the percentage of fluorescently labeled functionalized SWCNTs.

\section{Synthesis of SWCNT-lectin-FITC conjugates}

The functionalized SWCNTs were filtered and washed several times with deionized water, as before, until the $\mathrm{pH}$ of the solution was 6-7. The neutralized functionalized SWCNTs were dried. A volume of $1 \mathrm{~mL}$ of PBS was added to $1.2 \mathrm{mg}$ of functionalized SWCNTs; the solution was mixed with $6 \mathrm{mg}$ of EDC and $6 \mathrm{mg}$ NHS and placed on a rocker at room temperature for 30 minutes. A volume of $50 \mu \mathrm{L}$ of lectin-FITC $(20 \mu \mathrm{g} / \mathrm{mL})$ was added to the mixture and agitated for 2 hours on ice. A centrifugal column with a cutoff of $10 \mathrm{kDa}$ was used to remove the unbound material (Centricon, Millipore, UK).

\section{Cell culture}

Human breast cancer cell line MCF-7 (derived from a pleural effusion of a breast cancer patient) and human colon adenocarcinoma cell line (SW480) were grown in DMEM supplemented with 10\% (v/v) FCS (Biosera, East Sussex, UK). MCF-7 cells have been shown to exhibit a metastatic phenotype when implanted subcutaneously into SCID (severe combined immunodeficiency) mice.

Each cell type was grown to $80 \%$ confluence at $37^{\circ} \mathrm{C}$ under $5 \%(\mathrm{v} / \mathrm{v}) \mathrm{CO}_{2}$; for routine passage, the cells were washed three times with $10 \mathrm{mM}$ sodium phosphate buffer, $\mathrm{pH}$ 7.2 , containing $137 \mathrm{mM} \mathrm{NaCl}(\mathrm{PBS})$, incubated at $37^{\circ} \mathrm{C}$ with $0.5 \mathrm{~g} / \mathrm{L}$ trypsin and $0.2 \mathrm{~g} / \mathrm{L}$ EDTA (ethylenediaminetetraacetic acid) in PBS until they lifted from the flask, centrifuged at $400 \times g$ for 5 minutes and resuspended in fresh media. The resuspended pellet was passaged at a ratio of 1:6 into new flasks. Cells were also seeded on 12-well plates, with approximately 150,000 cells seeded into each well. To each well, $1 \mathrm{~mL}$ of treatment reagent was added.

\section{Cellular staining}

Lectin staining was performed as follows: 12-well plates containing MCF-7 and SW480 cells were washed three times with PBS and fixed for 30 minutes in $3.7 \%$ (v/v) formaldehyde, blocked for 30 minutes with $5 \%$ (w/v) BSA, trypsinized for 20 minutes at $37^{\circ} \mathrm{C}$, and incubated in the dark with $1 \mathrm{~mL}$ HPA-FITC at $20 \mu \mathrm{g} / \mathrm{mL}$ for 60 minutes in PBS. The cells were treated with $100 \mu \mathrm{g} / \mathrm{mL}$ DNase-free ribonuclease A for 20 minutes at $37^{\circ} \mathrm{C}$, and the nuclei counterstained using $1 \mu \mathrm{M}$ To-Pro-3 in PBS for 20 minutes. Cells were also incubated with SWCNTs conjugated to HPA-FITC (lectin-SWCNTs) using the same methodology and $20 \mu \mathrm{g} / \mathrm{mL}$ lectin-SWCNT.

Images were acquired by sequential scanning using a Leica TCS SP2 confocal system (Leica Microsystems, Milton Keynes, UK) with a $63 \mathrm{X}$ ceramic dipping objective at $1024 \times 1024$ format and a scanning speed of $400 \mathrm{~Hz}$, with a line average of 2 . A $488 \mathrm{~nm}$ laser, intensity $25 \%$, was used for excitation of the lectin-FITC; the emitted light was recorded over a bandwidth of 500-550 nm. A $633 \mathrm{~nm}$ laser, intensity $35 \%$, was used for To-Pro-3 excitation, with the emitted light recorded over a bandwidth of 650-720 nm.

\section{Assessment of the functionalization of SWCNT}

\section{Transmission electron microscopy (TEM)}

Functionalized SWCNTs and control material was deposited on a copper grid coated with Piloform (TAAB, Aldermaston, UK), and then TEM images (Phillips CM 120, Amsterdam, The Netherlands) were obtained and evaluated to investigate the efficiency of the functionalization process.

\section{Fourier transform infrared spectroscopy (FTIR)}

FTIR spectroscopy analysis was used to detect carboxylic acid groups $(-\mathrm{COOH})$ on SWCNTs using the PerkinElmer 
1750 FTIR (Beaconsfield, UK). The absorbance spectrum was obtained via a second derivative and a 13-point reading. The system utilizes a beam of light with many frequencies and measures the absorption spectrum of the sample. The group frequency wave numbers correspond to the functional groups that are present in the sample. This is, therefore, a useful technique for detecting the presence of chemical groups on a given sample. The FTIR was performed using two samples: non-functionalized SWCNTs and functionalized SWCNTs.

A $10 \mu \mathrm{L}$ volume of each of these samples, each $1 \mathrm{mg} / \mathrm{mL}$, was loaded onto the mounting crystal of the FTIR machine. The samples were prepared in $\mathrm{D}_{2} \mathrm{O}$ (heavy water) as the group frequency wave number/absorption peak of water would interfere with the absorption peak of - $\mathrm{COOH}$ groups $\left(3400 \mathrm{~cm}^{-1}\right)$, which the authors were trying to detect on the functionalized SWCNTs. The scan parameters were as follows: start $\left(4000 \mathrm{~cm}^{-1}\right)$, number of scans (100), resolution (4), units (\% $\%$, end (400 $\left.\mathrm{cm}^{-1}\right)$, interval (2), shuttle (interleaved).

\section{Analysis of functionalized CNTs using confocal microscopy}

The SWCNTs were treated with the acid solution for 0.5 , $1,2,3,4$, and 5 hours and subsequently attached to the HPA lectin-FITC as described above. The SWCNTs were viewed under a Leica scanning confocal microscope (Leica Microsystems). A $488 \mathrm{~nm}$ laser, intensity 25\%, was used for excitation, and the emitted light was recorded over a bandwidth of 500-550 nm. The material was also viewed using white light and this allowed the total number of SWCNTs to be determined, the percentage of SWCNTs that had been successfully conjugated with lectin-FITC was then calculated.

\section{Contact angle measurements to investigate surface hydrophobicity}

To evaluate the effect of the functionalization on the hydrophobicity of the SWCNTs, surface properties were studied pre- and post-functionalization using a drop-shape analysis system (DSA100, Hamburg, Germany). This enabled the steric contact angle measurement, which is referred to as $\theta$, to be determined using the sessile drop method. In this experiment the same quantity of functionalized and nonfunctionalized SWCNT powder was placed on the surface of solid graphite, which has the same chemical component as SWCNT. A sessile drop of water $(10 \mu \mathrm{L})$ was deposited on the surface of graphite functionalized and non-functionalized surfaces and the angle of water droplets on the surface of all materials was measured. The measurements were repeated at three different areas on each of the surfaces.

\section{Use of NIR light to radiate the SWCNTs}

Laser diodes of wavelengths $808 \mathrm{~nm}$ with maximum operating power of $1 \mathrm{~W}$ were purchased from Thorlabs Ltd along with other necessary equipment to operate the laser diode. Functionalized and non-functionalized SWCNTs were dispersed in PBS and heated until the dry powder (SWCNTs) remained. The samples were resuspended such that the concentration was $1 \mathrm{mg} / \mathrm{mL}$, centrifuged for 5 minutes at $3000 \times g$, and the supernatant retained; this was subjected to NIR irradiation. The temperature increase with respect to time of exposure was measured.

\section{Study of HPA lectin and HPA lectin- SWCNT interaction with human breast cancer cells (MCF-7)}

The Attana-200 ${ }^{\circledR}$ Biosensor (Attana AB, Stockholm, Sweden) is one of the most sophisticated machines in the area of molecular interaction analysis enabling studies of binding properties of bioconjugates at cell surface. This apparatus was used to investigate the association rate constant (rate of attachment) and dissociation of HPA-lectin and HPA-lectin conjugated to SWCNTs to human MCF-7 cells grown on the biosensor surface. The biosensor utilizes a quartz crystal microbalance (QCM) technique, enabling real-time, labelfree measurements of molecular interactions. When molecules are added to or removed from the surface, a change in the resonance frequency corresponds to the change in mass on the sensor surface. By immobilizing a target molecule to the sensor surface, and introducing an interacting molecule over the surface, the interaction can be studied in real-time. The real-time data can provide information on kinetics, affinity, active concentration, and specificity for the interaction.

Cell culture-compatible polystyrene-coated QCM sensor chips were obtained from Attana AB. The sensor chip was placed face-up in a well of a 24-well plate. An appropriate dilution of MCF-7 cells (to obtain 40,000 cells on the sensor surface) was added in $2 \mathrm{~mL}$ of media to the well, and the plate was placed in an incubator at $37^{\circ} \mathrm{C}\left(5 \%(\mathrm{v} / \mathrm{v}) \mathrm{CO}_{2}\right.$ and $95 \%$ humidity) for 20 hours. Following incubation, the cells were washed three times with PBS and fixed for 30 minutes in fresh $3.7 \%(\mathrm{v} / \mathrm{v})$ formaldehyde in PBS. All experiments were performed using an Attana Cell 100 or Attana Cell 200 biosensor (Attana AB). The running buffer was PBS with $0.025 \%$ (v/v) Tween-20. The cell chip was fitted to the machine and allowed to stabilize overnight at $20^{\circ} \mathrm{C}$ at a flow rate of $25 \mu \mathrm{L} / \mathrm{min}$. HPA lectin $(12.5 \mu \mathrm{g} / \mathrm{mL}$, 
$25 \mu \mathrm{g} / \mathrm{mL})$ or HPA lectin-SWCNTs $(12.5 \mu \mathrm{g} / \mathrm{mL}, 25 \mu \mathrm{g} / \mathrm{mL}$ HPA conjugated to SWCNT at $100 \mu \mathrm{g} / \mathrm{mL}$ ) was injected over the surface of the chip at a range of concentrations and allowed to bind for 85 seconds. The injection was then stopped, and the dissociation of the lectin was followed for 165 seconds. Following each sample injection cycle (association and dissociation), the cell chip was regenerated using two 30 -second pulses of $10 \mathrm{mM}$ glycine, $\mathrm{pH}$ 1.0, $0.5 \mathrm{M} \mathrm{NaCl}$, followed by immediate re-equilibration with appropriate running buffer.

\section{Statistical analysis}

Statistical significance testing was undertaken using the Student's $t$-test (paired). $P$-values of $<0.05$ were considered statistically significant.

\section{Results}

\section{Preparation of functionalized SWCNT}

Treatment of SWCNT with a combination of $\mathrm{HNO}_{3}$ and $\mathrm{H}_{2} \mathrm{SO}_{4}$ resulted in successful attachment of $-\mathrm{COOH}$ over the surface of the material. Different methods, such as TEM, FTIR, confocal microscopy, contact angle measurements and NIR wavelength were used to establish successful functionalization of the SWCNTs, as described below.

The functionalization led to an increase in the amount of dispersion of the SWCNTs, and 2 weeks post-functionalization, the SWCNTs remained dispersed in solution (Figure 1). Following functionalization, both the internal and external diameter of the nanotubes increased, whilst the length of the SWCNTs decreased (Table 1).

\section{TEM as a tool for investigating the surface topography of SWCNT}

TEM was used to investigate the pristine SWCNTs and the SWCNTs treated with $\mathrm{HNO}_{3}: \mathrm{H}_{2} \mathrm{SO}_{4}$. In this analysis, the functionalized SWCNTs were observed to have acquired a thick black layer covering their surfaces (Figure 1).

\section{Synthesis of SWCNT-lectin-FITC conjugates}

Lectins are carbohydrate-binding proteins that show specificity for particular glycan moieties. They are known to be of utility in the recognition of cancer cells, as changes in glycosylation are a feature of tumorigenesis and relate to the metastatic behavior of cancer cells. ${ }^{31}$ EDC and NHS were used as coupling agents to attach FITC-labeled HPA lectin to the SWCNTs. Following the addition of the lectin-FITC, the SWCNTs were viewed using the confocal microscope. The SWCNTs emitted light in the green spectra consistent with attachment of FITC-labeled material (Figure 2). When the SWCNT were exposed to acid for $>2$ hours a statistically significant increase in attachment of lectin-FITC was observed compared with only 0.5 hours exposure (Figure 3 ).

\section{FTIR spectroscopy}

FTIR analysis was performed in order to verify that a $-\mathrm{COOH}$ group had been attached to the SWCNTs during the functionalization process. The FTIR spectra obtained for the functionalized SWCNTs clearly revealed peaks at $1580 \mathrm{~cm}^{-1}, 1750 \mathrm{~cm}^{-1}$, and $3450 \mathrm{~cm}^{-1}$ (Figure 4A), but for the pristine SWCNTs, only a peak at $1580 \mathrm{~cm}^{-1}$ was recorded (Figure $4 \mathrm{~B}$ ).

\section{Lectin-SWCNTs binding to MCF-7 breast cancer cells}

MCF-7 cells were incubated with the HPA-FITC alone and with lectin-SWCNTs and viewed using confocal microscopy (Figure 5). Cell surface localization of HPA was observed; this is consistent with the recognition of $\mathrm{N}$-acetylgalactosamine containing glycoproteins as reported in previous work from

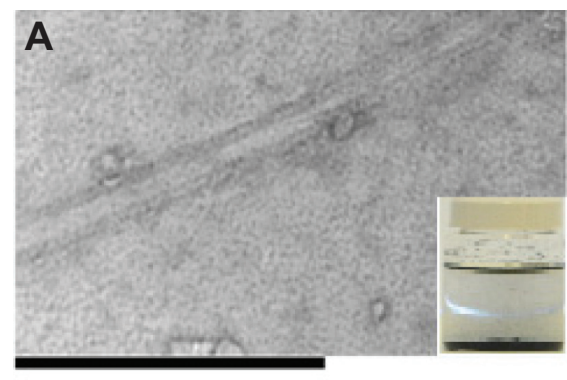

$100 \mathrm{~nm}$

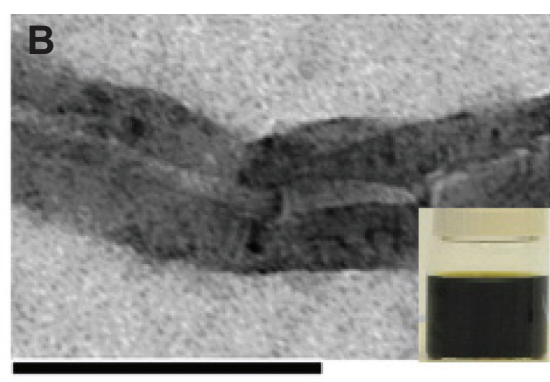

$100 \mathrm{~nm}$

Figure I Transmission electron microscopy of SWCNTs pre- and post-functionalization. SWCNTs before $(\mathbf{A})$ and after $(\mathbf{B})$ oxidization using $\mathrm{HNO}_{3}: \mathrm{H}_{2} \mathrm{SO}_{4}$. Treatment with acid resulted in chemical modifications of the SWCNTs and the formation of carboxyl groups on the surface. Inset: tube containing SWCNTs, showing the enhancement of dispersibility of SWCNTs 2 weeks post-functionalization. Abbreviation: SWCNT, single-walled carbon nanotube. 
Table I Physical properties of pre- and post-functionalized SWCNTs

\begin{tabular}{lccl}
\hline $\begin{array}{l}\text { Physical properties } \\
\text { of SWCNTs }\end{array}$ & \multicolumn{2}{l}{ Functionalization } & P-value \\
\cline { 2 - 3 } & $\begin{array}{l}\text { Pre } \\
\text { (mean } \pm \text { SD) }\end{array}$ & $\begin{array}{l}\text { Post } \\
\text { (mean } \pm \text { SD) }\end{array}$ & \\
\hline Internal diameter $(\mathrm{nm})$ & $9.6 \pm 1.14$ & $1 \mathrm{I} .60 \pm 1.14$ & $<0.05$ \\
External diameter $(\mathrm{nm})$ & $13.20 \pm 1.90$ & $46 \pm 3.50$ & $<0.0 \mathrm{I}$ \\
Length $(\mathrm{nm})$ & $350 \pm 79.05$ & $250 \pm 61.07$ & 0.07 \\
Contact angle $(\theta)$ & $86.1 \pm 0.60$ & $18.5 \pm 0.63$ & $<0.05$ \\
\hline
\end{tabular}

Notes: Both the internal and external diameters of SWCNTs increase after functionalization due to the attachment of $-\mathrm{COOH}$ groups. However, the covalent functionalization shortens the length of SWCNTs.

Abbreviations: SD, standard deviation; SWCNT, single-walled carbon nanotube.

the authors of this present paper and others. ${ }^{11}$ The cells incubated with lectin-SWCNTs showed a more intense staining pattern than those incubated with HPA alone; it is presumed that this is due to attachment of a multitude of FITC-labeled lectin molecules to each SWCNT structure.

\section{Contact angle analysis}

Pre-functionalization, the contact angle was $86.1^{\circ} \pm 0.60^{\circ}$. The contact angle on the surface of graphite was determined as $89.5^{\circ} \pm 1.5^{\circ}$, thereby indicating the hydrophobic nature of the material pre-functionalization. Post-functionalization, the contact angle measurements dramatically reduced to $18.5^{\circ} \pm 0.63^{\circ}$ (Table 1$)$.

\section{NIR wavelength analysis}

NIR irradiation of a solution containing dispersed functionalized SWCNTs using laser light $(\lambda 808 \mathrm{~nm})$ for 10 minutes resulted in an increase in temperature of $24.5^{\circ} \mathrm{C}$, equivalent to a $2.4^{\circ} \mathrm{C}$ rise per minute exposure at $808 \mathrm{~nm}$. In contrast, NIR irradiation of a solution containing dispersed but non-functionalized SWCNTs at the same wavelength of light and over the same period produced a rise in temperature of only $4.4^{\circ} \mathrm{C}$, equivalent to a $0.4^{\circ} \mathrm{C}$ rise in temperature per minute (Figure 6).

\section{The affinity of lectin-SWCNTs to human breast cancer cells}

The binding properties of HPA lectin conjugated to SWCNTs to glycan epitopes on the surface of the metastatic breast cancer cell line, MCF-7, was assessed using a novel cell biosensor. In this system, the on-rate and off-rate of biomolecules are assessed at the cell surface. The interaction between the HPA-lectin as well as the lectin-SWCNT conjugate at the cancer cell surface was measured and was observed to increase in a dose-dependent manner, as measured by the change in the resonance frequency $(\Delta \mathrm{F})$. The affinity of the lectin HPA to the MCF-7 cells, the dissociation rate $\left(\mathrm{K}_{\mathrm{D}}\right)$, was in the nanomolar range. The sensorgrams indicated that the lectin interaction at the cell surface of MCF-7 cells is not affected by attachment of HPA to the SWCNTs (Figure 7).

\section{Discussion}

Pristine SWCNTs are severely contaminated with metal catalysts and amorphous carbons. They are also insoluble and as such this poses problems for their use in a cellular environment. ${ }^{32,33}$ Functionalization is a technique used to overcome this issue. The functionalization process involves the attachment of other materials to the sides or ends of the SWCNTs in order to enhance their biocompatibility; this may

B

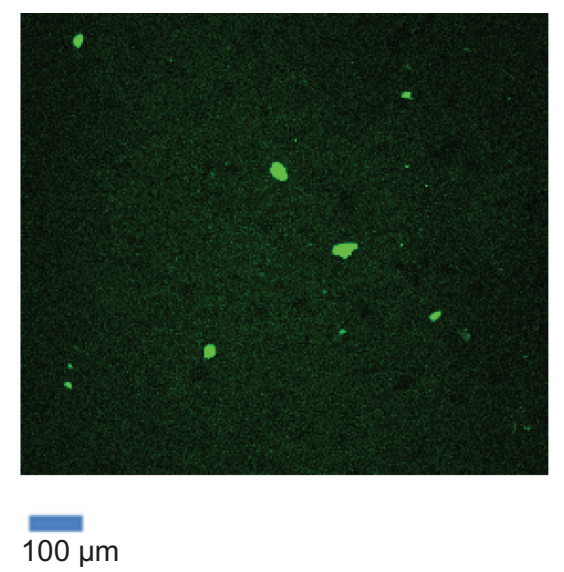

Figure 2 HPA-lectin-FITC-SWCNTs. A sample of functionalized SWCNTs conjugated to HPA-FITC was viewed under the confocal microscope, in transmission mode (A) and in fluorescence mode, following excitation at $488 \mathrm{~nm}$ (B). The results show that a proportion of the SWCNTs had been successfully conjugated to the FITC-labeled lectin. Abbreviations: FITC, fluorescein isothiocyanate; HPA, Helix pomatia agglutinin; SWCNT, single-walled carbon nanotube. 


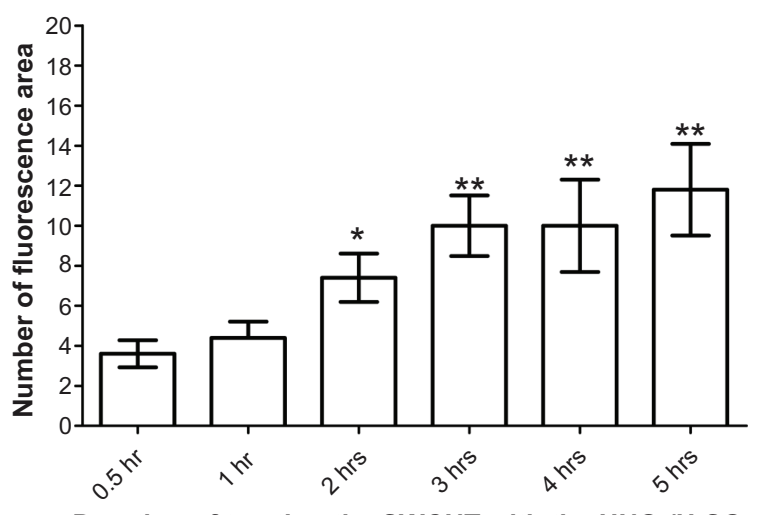

Duration of treating the SWCNT with the $\mathrm{HNO}_{3} / \mathrm{H}_{2} \mathrm{SO}_{4}$

Figure 3 The effect of different acid exposure times on attachment of lectin to SWCNTs. The SWCNTs were treated with acid for 0.5, I, 2, 3, 4, and 5 hours, followed by HPA-lectin-FITC conjugation. Confocal microscopy was used to determine the relative fluorescence for the SWCNTs prepared. An increase in the fluorescence was observed as the exposure time of SWCNTs to $\mathrm{HNO}_{3}$ and $\mathrm{H}_{2} \mathrm{SO}_{4}$ increased. The relative fluorescence following 0.5 hours treatment with $\mathrm{HNO}_{3}$ and $\mathrm{H}_{2} \mathrm{SO}_{4}$ was compared with longer incubation periods.

Notes: Statistical analysis was performed using the Student's $t$-test, and the $P$-values are indicated. Values are mean \pm standard deviation. $* P<0.05(2 \mathrm{hrs}) ; * P<0.01$ ( $3 \mathrm{hrs}, 4 \mathrm{hrs}$ and 5 hrs).

Abbreviations: FITC, fluorescein isothiocyanate; HPA, Helix pomatia agglutinin; SWCNT, single-walled carbon nanotube.

be achieved via covalent or noncovalent bonding. ${ }^{34}$ Each type of bonding has unique advantages and disadvantages. For example, the covalent bonding of a functional group to the SWCNTs will produce stable functionalized SWCNTs making the material suitable for a multitude of applications such as drug delivery. ${ }^{33}$ One of the major problems, however, with the SWCNTs is their lack of solubility and an insufficient number of functional groups for binding to other biomolecules; consequently problems may arise if these materials were to be used for diagnosis and treatment of cancer. Coating a SWCNT with different biocompatible materials would alleviate this problem; one way of coating the SWCNTs would be by treating the SWCNTs with acid. ${ }^{2}$

In this manuscript, the treatment of SWCNTs with a combination of acids was investigated. The system was used to coat the surface of the SWCNT resulting in the attachment of a carboxylic acid group $(-\mathrm{COOH})$, thereby producing a dispersable, hydrophilic SWCNT. Carboxylation of SWCNTs is a common method for the covalent functionalization of SWCNTs, as the preparation method is relatively simple and quick. The oxidation process does not require complex apparatus and can be undertaken in a short period of time. Another benefit of carboxylation is that the carboxylic group can target other functional groups such as amines or thiols. It is important to emphasize that whilst carboxylation has many benefits, the physical properties of the SWCNTs are altered during the functionalization process; therefore, it is recommended that in-vitro experiments are undertaken prior to assessment of the suitability of the material using an invivo approach.

In this study it was observed that after the SWCNTs had been treated with acid, the particles remained dispersed in PBS after 2 weeks; in contrast, the pristine SWCNTs were found as a precipitate and no longer dispersed in the PBS. This suggests that treating SWCNTs with $\mathrm{HNO}_{3}: \mathrm{H}_{2} \mathrm{SO}_{4}$ increases the hydrophilic nature of the SWCNTs and potential biocompatibility.

TEM analysis showed that a thick black layer had formed on the surface of the SWCNTs following treatment with acid. A significant increase in the external and internal diameter was also detected. The difference of increased external diameter, in comparison to internal diameter, indicating successful attachment of the functional groups to the outer surface of the

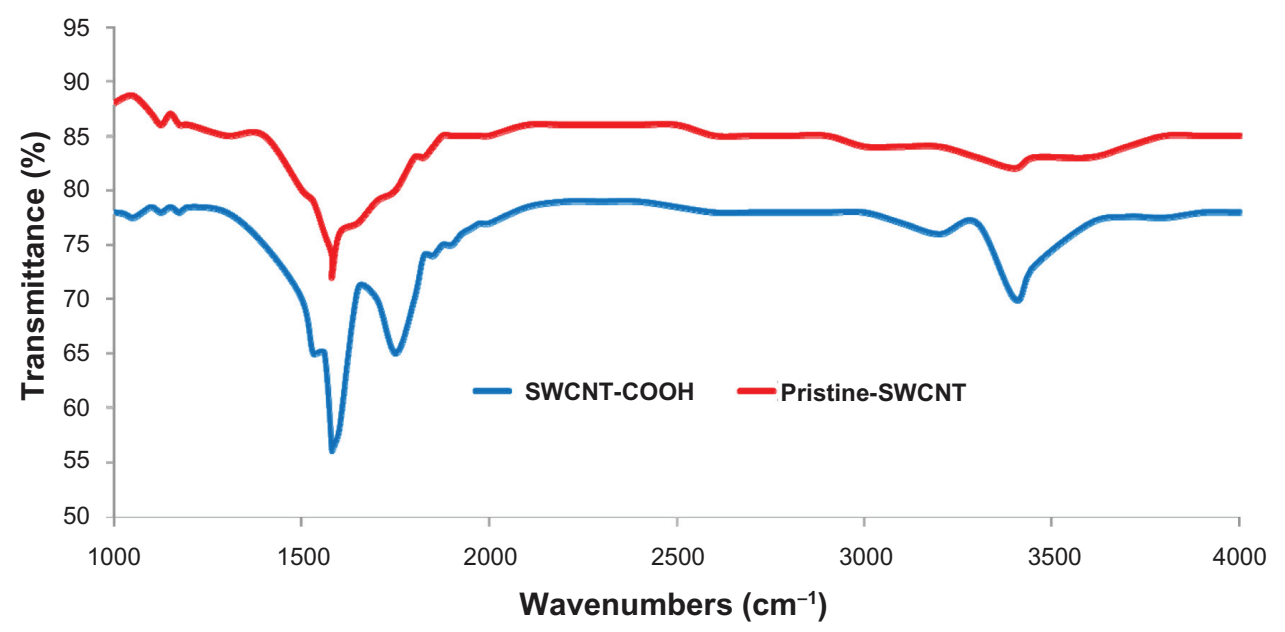

Figure 4 Fourier transform infrared spectroscopy analysis of SWCNTs. Before (red) and after (blue) functionalization. Following functionalization, peaks observed at $1750 \mathrm{~cm}^{-1}$ and $3450 \mathrm{~cm}^{-1}$ indicate that $-\mathrm{COOH}$ groups had been successfully grafted onto the surface of the SWCNTs.

Abbreviation: SWCNT, single-walled carbon nanotube. 
MCF7

To-Pro-3
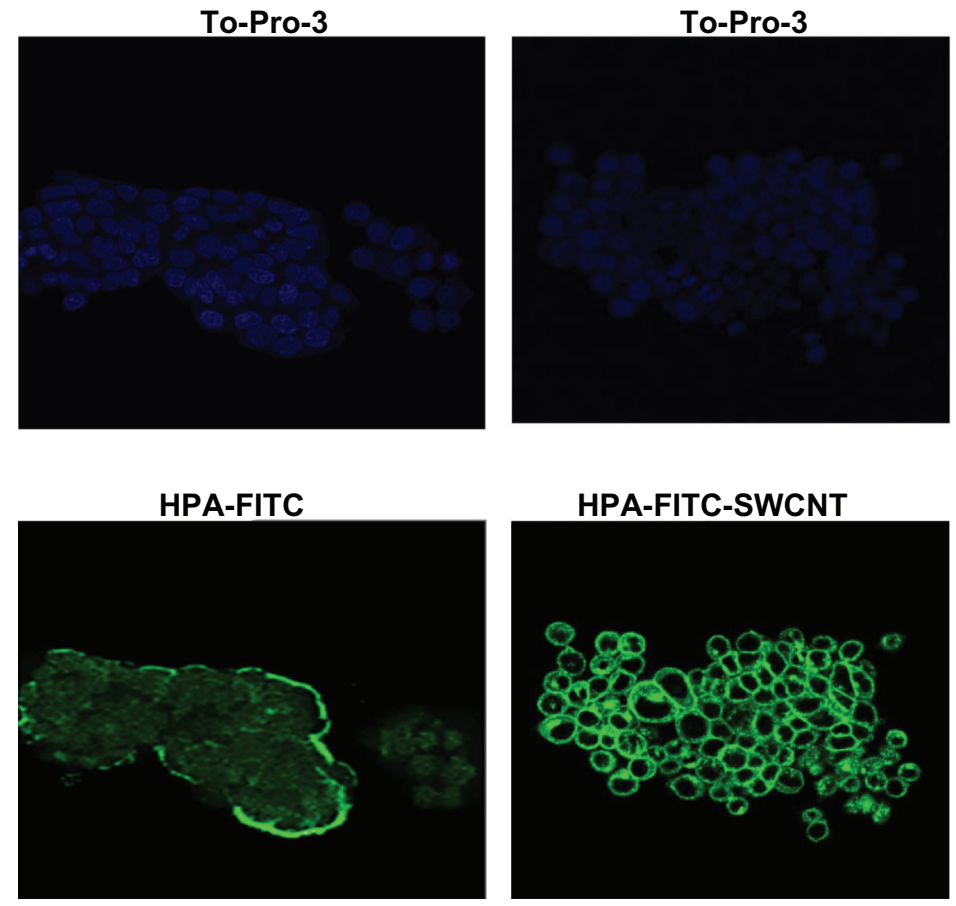

Figure 5 Confocal microscopy of MCF-7 breast cancer cells incubated with HPA-FITC and HPA-lectin-FITC linked to SWCNTs. Lectin binding to MCF-7 and SW480 cells as viewed using a Leica TCS SP2 confocal microscope. Cells were incubated with FITC-labeled HPA (green) and lectin-SWCNTs (green), and the nuclei were counterstained with To-Pro ${ }^{\circledR 3}$ (blue). MCF-7 were observed to interact with the HPA lectin either linked to FITC or to SWCNT; in contrast, the SW480 cells did not bind the lectin. Abbreviations: FITC, fluorescein isothiocyanate; HPA, Helix pomatia agglutinin; SWCNT, single-walled carbon nanotube.

SWCNTs. The results also showed that treatment with acid decreased the overall size of the SWCNTs. This will enhance the reaction sites of the SWCNTs with other reagents such as antibodies and drugs.

FITR was also undertaken. The spectra showed a peak at the $1580 \mathrm{~cm}^{-1}$ region for both the functionalized

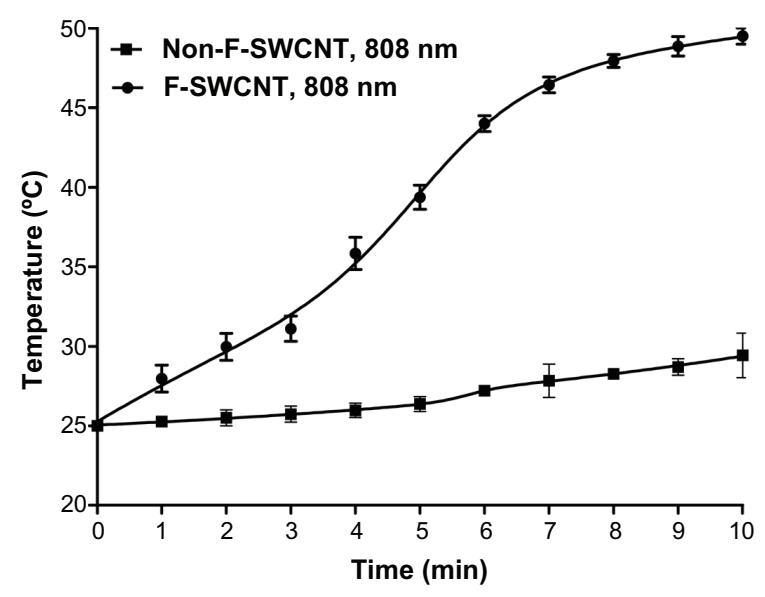

Figure 6 Temperature profile of SWCNTs, pre- and post-functionalization. Following exposure to light at $\lambda 808 \mathrm{~nm}$, functionalized SWCNTs achieved a higher temperature profile compared with non-functionalized CNTs.

Note: Mean values \pm standard deviation are shown.

Abbreviations: CNT, carbon nanotube; SWCNT, single-walled CNT; F-SWCNT, functionalized SWCNT. and non-functionalized SWCNTs; this is attributed to the $\mathrm{C}=\mathrm{C}$ stretching vibration. However, additional peaks were observed at $1750 \mathrm{~cm}^{-1}$ and $3450 \mathrm{~cm}^{-1}$ for functionalized SWCNT spectra. The band at $1750 \mathrm{~cm}^{-1}$ is due to carboxyl $(\mathrm{C}=\mathrm{O})$ stretching vibration, and the band at $3450 \mathrm{~cm}^{-1}$ is assigned to the $(-\mathrm{OH})$ stretching vibration of the carboxylic acid group. These values clearly illustrate that the $-\mathrm{COOH}$ groups were successfully grafted onto the surface of the functionalized SWCNTs.

Contact angle measurements showed that after treating the SWCNTs with acid, the $\theta$ values decreased dramatically; this was indicative of the chemical and structural changes that occurred at the surface of the SWCNTs, hence the hydrophilicity of the SWCNTs increased.

One of the main properties of SWCNTs is their ability to absorb NIR wavelength light and generate heat. This property of the SWCNTs was used to establish whether the functionalization process had been successful. According to the authors' hypothesis, the SWCNT particles that were processed with acid would remain dispersed in water (in the supernatant) following centrifugation. Based on this hypothesis, and that a key property of SWCNTs is their ability to absorb light in the NIR spectra and generate heat, the supernatant of both functionalized and non-functionalized 

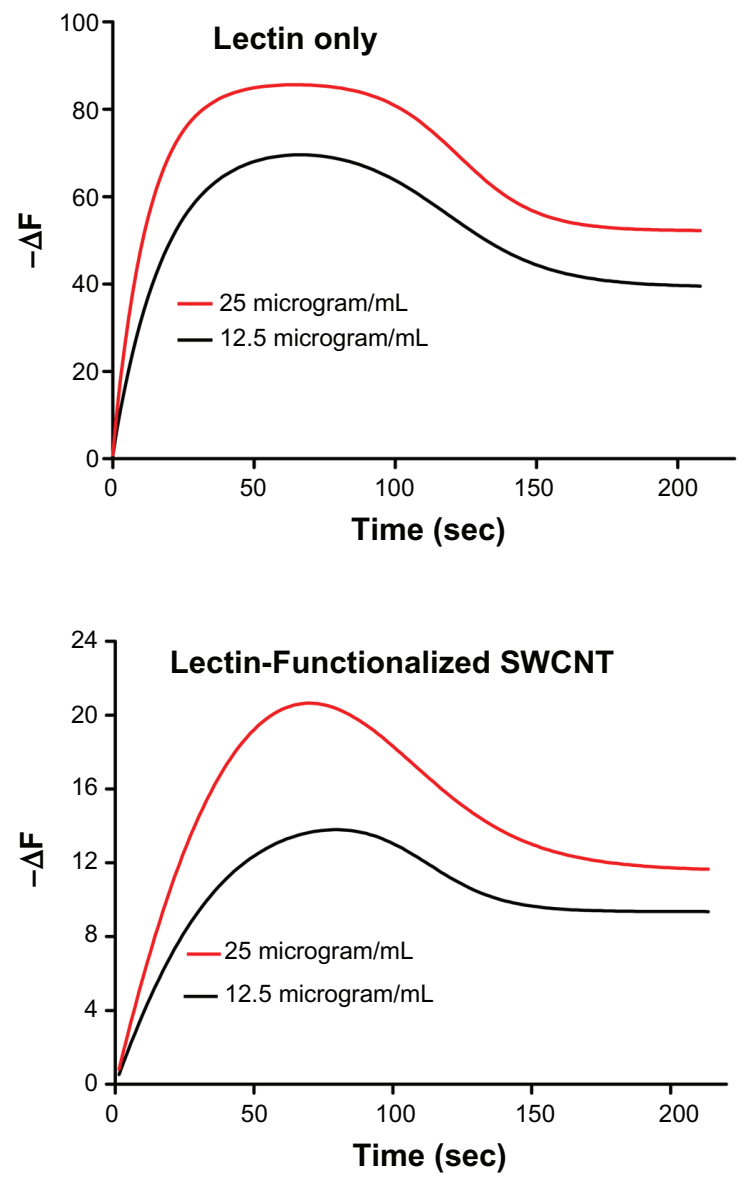

Figure 7 Sensorgrams illustrating the binding profile of HPA-FITC and HPA-lectinSWCNTs to MCF-7 cells grown on a biosensor chip surface. The association (85 second) and dissociation ( 165 second) phases were monitored giving a change in the resonance frequency $(\Delta F)$ as the lectin interacted with the surface of the MCF-7 cells on the chip surface. The lectin interaction with MCF-7 cells was investigated at $12.5 \mu \mathrm{g} / \mathrm{mL}$ and $25 \mu \mathrm{g} / \mathrm{mL}$ as shown, using HPA alone and HPA-lectinSWCNTs. The flow rate was maintained at $25 \mu \mathrm{L} / \mathrm{min}$, and the temperature was $22^{\circ} \mathrm{C}$. The binding profile for native HPA was comparable to the profile obtained for HPA-lectin-SWCNTs, indicating that the conjugation process did not affect the rate of epitope binding by the lectin.

Abbreviations: FITC, fluorescein isothiocyanate; HPA, Helix pomatia agglutinin SWCNT, single-walled carbon nanotube.

samples was treated using $808 \mathrm{~nm}$ wavelength light. As expected, the supernatant from functionalized SWCNTs showed a greater temperature increase as compared with pristine SWCNTs.

Finally, confocal microscopy was used to establish successful functionalization of the SWCNTs. Following treatment of pristine SWCNTs with acid, the material was reacted with HPA lectin-FITC and viewed under the confocal microscope. It was predicted that as the exposure time of SWCNTs to acid increased, more- $\mathrm{COOH}$ groups should be present on the surface of the SWCNTs and that this would in turn result in an increase in the amount of HPA lectin-FITC attached. The observed increase in the lectin-FITC signal, as exposure of the SWCNTs to acid increased, was consistent with this hypothesis.
To check that the attachment of HPA to the SWCNTs did not adversely affect the protein structure and function, the interaction between HPA lectin-FITC conjugated to SWCNTs (and HPA lectin-FITC alone) was assessed using the human breast cancer cell line MCF-7. The analysis showed that HPAFITC as well as HPA lectin-FITC-SWCNTs both bind to the cell membrane of MCF-7, indicating that the attachment process to SWCNTs did not adversely affect glycan-lectin interactions. To explore this further and to evaluate the potential for delivering SWCNTs to human breast cancer cells, the binding of the lectin to the cell surface of MCF-7 cells was explored using a cell biosensor system. Here, the lectin is flowed over the cell surface, and the attachment (on-rate) and detachment (off-rate) is monitored using a quartz crystal microbalance and this allows the affinity $\left(\mathrm{K}_{\mathrm{D}}\right)$ of the molecule to the cell surface to be determined. The results showed that when HPA was attached to the SWCNTs, the conjugate bound to the surface of MCF-7 cells in a manner comparable to the nonconjugated lectin. On the basis of these results, it can be concluded that HPA lectin is a promising candidate for the in-vitro delivery of SWCNTs to human breast cancer cells.

\section{Conclusion}

SWCNTs have potential application in new cancer therapies, particularly when the drug delivery capacity and their ability to absorb NIR radiation are considered. Efficient functionalization of the SWCNTs is, however, a crucial step prior to using these materials for drug loading/delivery to cancer cells. One way to achieve viable functionalization of SWCNT is to treat the materials with $\mathrm{HNO}_{3}: \mathrm{H}_{2} \mathrm{SO}_{4}(1: 3)$ $\left[\mathrm{HNO}_{3}(70 \%) / \mathrm{H}_{2} \mathrm{SO}_{4}(95 \%-98 \%)\right]$ and reflux for 120 minutes at $120^{\circ} \mathrm{C}$. Treating the SWCNT resulted in the formation of a carboxylic acid group on the surface giving a hydrophilic structure. The presence of the carboxylic groups enables the attachment of molecules such as antibodies, glycoproteins, lectins, and carbohydrates, allowing the SWCNTs to be used to specifically target cancer cells. As changes in glycosylation are a feature of cancer development and progression, this allows the selection of an appropriate biomolecule such as HPA lectin for conjugation to SWCNTs in order to specifically target cancer cells.

\section{Acknowledgments}

This study was supported by a grant by Technology Strategy Board, UK, and Engineering and Physical Science Research Council (EPSRC), UK. The authors thank Dr D Peiris, University of Westminster for assistance in using confocal microscopy and using Attana Biosensor for cell interaction 
study, Dr B Cousin for assistance in contact angle measurement, Dr B Ramesh and Mr Darbyshire for assistance in FTIR and spectroscopy of carbon nanotubes.

\section{Disclosure}

The authors report no conflicts of interest in this work. This paper was presented at the Society of Academic and Research Surgery Conference (SARS), Dublin, Ireland, in January 2011.

\section{References}

1. Tan A, Yildirimer L, Rajadas J, De La PH, Pastorin G, Seifalian A. Quantum dots and carbon nanotubes in oncology: a review on emerging theranostic applications in nanomedicine. Nanomedicine (Lond). 2011;6(6):1101-1114.

2. Klumpp C, Kostarelos K, Prato M, Bianco A. Functionalized carbon nanotubes as emerging nanovectors for the delivery of therapeutics. Biochim Biophys Acta. 2006;1758(3):404-412.

3. Yu MF, Lourie O, Dyer MJ, Moloni K, Kelly TF, Ruoff RS. Strength and breaking mechanism of multiwalled carbon nanotubes under tensile load. Science. 2000;287(5453):637-640.

4. Shi X, Wang SH, Shen M, et al. Multifunctional dendrimer-modified multiwalled carbon nanotubes: synthesis, characterization, and in vitro cancer cell targeting and imaging. Biomacromolecules. 2009;10(7):1744-1750.

5. Wang N, Tang ZK, Li GD, Chen JS. Single-walled 4A carbon nanotube arrays. Nature. 2000;408(6808):50-51.

6. Dai H. Carbon nanotubes: synthesis, integration, and properties. Acc Chem Res. 2002;35(12):1035-1044.

7. Smart SK, Cassady AI, Lu GQ, Martin DJ. The biocompatibility of carbon nanotubes. Carbon. 2006;44(6):1034-1047.

8. Valentin N. Carbon nanotubes: properties and application. Mater Sci Eng R Rep. 2004;43(3):61-102.

9. Zhou F, Xing D, Ou Z, Wu B, Resasco DE, Chen WR. Cancer photothermal therapy in the near-infrared region by using single-walled carbon nanotubes. J Biomed Opt. 2009;14(2):021009.

10. Kam NW, Dai H. Carbon nanotubes as intracellular protein transporters: generality and biological functionality. J Am Chem Soc. 2005;127(16): 6021-6026.

11. Zhang Y, Bai Y, Yan B. Functionalized carbon nanotubes for potential medicinal applications. Drug Discov Today. 2010;15(11-12): 428-435.

12. Aliev AE, Lima MH, Silverman EM, Baughman RH. Thermal conductivity of multi-walled carbon nanotube sheets: radiation losses and quenching of phonon modes. Nanotechnology. 2010;21(3):035709.

13. Gottesman MM, Fojo T, Bates SE. Multidrug resistance in cancer: role of ATP-dependent transporters. Nat Rev Cancer. 2002;2(1):48-58.

14. Bianco A, Kostarelos K, Partidos CD, Prato M. Biomedical applications of functionalised carbon nanotubes. Chem Commun (Camb). 2005;(5):571-577.

15. Rippel RA, Seifalian AM. Gold revolution - gold nanoparticles for modern medicine and surgery. J Nanosci Nanotechnol. 2011;11(5): $3740-3748$.

International Journal of Nanomedicine

\section{Publish your work in this journal}

The International Journal of Nanomedicine is an international, peerreviewed journal focusing on the application of nanotechnology in diagnostics, therapeutics, and drug delivery systems throughout the biomedical field. This journal is indexed on PubMed Central, MedLine, CAS, SciSearch $\AA$, Current Contents ${ }^{\circledR} /$ Clinical Medicine,
16. de MA, Murad F, Seifalian AM. Nitric oxide: a guardian for vascular grafts? Chem Rev. 2011;111(9):5742-5767.

17. Tan A, De La PH, Seifalian AM. The application of exosomes as a nanoscale cancer vaccine. Int J Nanomedicine. 2010;5:889-900.

18. Coccini T, Roda E, Sarigiannis DA, et al. Effects of water-soluble functionalized multi-walled carbon nanotubes examined by different cytotoxicity methods in human astrocyte D384 and lung A549 cells. Toxicology. 2010;269(1):41-53.

19. Islam MF, Rojas E, Bergey DM, Johnson AT. High weight fraction surfactant solubilization of single-wall carbon nanotubes in water. Nano Lett. 2002;3:269-273.

20. Ghosh S, Dutta S, Gomes E, et al. Increased heating efficiency and selective thermal ablation of malignant tissue with DNA-encased multiwalled carbon nanotubes. ACS Nano. 2009;3(9):2667-2673.

21. Madani SY, Naderi N, Dissanayake O, Tan A, Seifalian AM. A new era of cancer treatment: carbon nanotubes as drug delivery tools. Int $J$ Nanomedicine. 2011;6:2963-2979.

22. Liu Z, Tabakman S, Welsher K, Dai H. Carbon nanotubes in biology and medicine: in vitro and in vivo detection, imaging and drug delivery. Nano Res. 2009;2(2):85-120.

23. Liu Z, Sun X, Nakayama-Ratchford N, Dai H. Supramolecular chemistry on water-soluble carbon nanotubes for drug loading and delivery. ACS Nano. 2007;1(1):50-56.

24. Liu Z, Tabakman SM, Chen Z, Dai H. Preparation of carbon nanotube bioconjugates for biomedical applications. Nat Protoc. 2009;4(9): 1372-1382.

25. Chen RJ, Zhang Y, Wang D, Dai H. Noncovalent sidewall functionalization of single-walled carbon nanotubes for protein immobilization. J Am Chem Soc. 2001;123(16):3838-3839.

26. Lay CL, Liu HQ, Tan HR, Liu Y. Delivery of paclitaxel by physically loading onto poly(ethylene glycol) (PEG)-graft-carbon nanotubes for potent cancer therapeutics. Nanotechnology. 2010;21(6):065101.

27. Dyke CA, Tour JM. Overcoming the insolubility of carbon nanotubes through high degrees of sidewall functionalization. Chemistry. 2004;10(4):812-817.

28. Ghanbari H, de Mel A, Seifalian AM. Cardiovascular application of polyhedral oligomeric silsesquioxane nanomaterials: a glimpse into prospective horizons. Int J Nanomedicine. 2011;6:775-786.

29. Kidane AG, Burriesci G, Edirisinghe M, Ghanbari H, Bonhoeffer P, Seifalian AM. A novel nanocomposite polymer for development of synthetic heart valve leaflets. Acta Biomater. 2009;5(7): 2409-2417.

30. Bianco A, Kostarelos K, Prato M. Applications of carbon nanotubes in drug delivery. Curr Opin Chem Biol. 2005;9(6):674-679.

31. Dwek MV, Ross HA, Streets AJ, et al. Helix pomatia agglutinin lectin-binding oligosaccharides of aggressive breast cancer. Int J Cancer. 2001;95(2):79-85.

32. Prato M, Kostarelos K, Bianco A. Functionalized carbon nanotubes in drug design and discovery. Acc Chem Res. 2008;41(1):60-68.

33. Bekyarova E, Ni Y, Malarkey EB, et al. Applications of carbon nanotubes in biotechnology and biomedicine. J Biomed Nanotechnol. 2005;1(1):3-17.

34. Niyogi S, Hamon MA, Hu H. Chemistry of single-walled carbon nanotubes. Acc Chem Res. 2011;35:1105-1113.

Journal Citation Reports/Science Edition, EMBase, Scopus and the Elsevier Bibliographic databases. The manuscript management system is completely online and includes a very quick and fair peer-review system, which is all easy to use. Visit http://www.dovepress.com/ testimonials.php to read real quotes from published authors. 